\title{
Microbial associations and spatial proximity predict North American moose (Alces alces) gastrointestinal community composition
}

Nicholas M. Fountain-Jones* ${ }^{1}$, Nicholas J. Clark ${ }^{2}$, Amy C. Kinsley ${ }^{1,4}$, Michelle Carstensen $^{3}$, James Forester ${ }^{5}$, Timothy J. Johnson ${ }^{4}$, Elizabeth Miller ${ }^{1}$, Seth Moore ${ }^{6}$, Tiffany M. Wolf ${ }^{1} \&$ Meggan E. Craft ${ }^{1}$.

${ }^{1}$ Department of Veterinary Population Medicine, University of Minnesota, St Paul, Minnesota 55108.

${ }^{2}$ UQ Spatial Epidemiology Laboratory, School of Veterinary Science, the University of Queensland, Gatton 4343, Queensland, Australia

${ }^{3}$ Minnesota Department of Natural Resources, Wildlife Health Program, 5463 West Broadway, Forest Lake, Minnesota, 55025, USA

${ }^{4}$ Department of Veterinary and Biomedical Sciences, University of Minnesota, St Paul, Minnesota 55108.

${ }^{5}$ Department of Fisheries, Wildlife and Conservation Biology, University of Minnesota, St Paul, Minnesota 55408, USA.

${ }^{6}$ Department of Biology and Environment, Grand Portage Band of Chippewa, Grand Portage, MN 55605

* Department of Veterinary Population Medicine, University of Minnesota, 1365 Gortner Avenue, St Paul, Minnesota 55108.nfj@umn.edu 


\section{Abstract}

1. Microbial communities are increasingly recognised as crucial for animal health.

However, our understanding of how microbial communities are structured across wildlife populations is poor. Mechanisms such as interspecific associations are important in structuring free-living communities, but we still lack an understanding of how important interspecific associations are in structuring gut microbial communities in comparison to other factors such as host characteristics or spatial proximity of hosts.

2. Here we ask how gut microbial communities are structured in a population of North American moose (Alces alces). We identify key microbial interspecific associations within the moose gut and quantify how important they are relative to key host characteristics, such as body condition, for predicting microbial community composition.

3. We sampled gut microbial communities from 55 moose in a population experiencing decline due to a myriad of factors, including pathogens and malnutrition. We examined microbial community dynamics in this population utilizing novel graphical network models that can explicitly incorporate spatial information.l

4. We found that interspecific associations were the most important mechanism structuring gut microbial communities in moose and detected both positive and negative associations. Models only accounting for associations between microbes had higher predictive value compared to models including moose sex, evidence of previous pathogen exposure, or body condition. Adding spatial information on moose location further 
strengthened our model and allowed us to predict microbe occurrences with 90\% accuracy.

5. Collectively, our results suggest that microbial interspecific associations coupled with host spatial proximity are vital in shaping infra communities in a large herbivore. In this case, previous pathogen exposure and moose body condition were not as important in predicting gut microbial community composition. The approach applied here can be used to quantify interspecific associations and gain a more nuanced understanding of the spatial and host factors shaping microbial communities in non-model hosts.

Key words: Biotic interactions, Body condition, Co-occurrence networks, Markov random fields, Microbiome, Pathogens, Spatial analysis 


\section{Introduction}

2

3 The relative roles of interspecific associations versus the environment in shaping

4 communities are intensely debated (e.g., Chase \& Myers, 2011; Vellend et al., 2014). In

5 particular, detecting negative (e.g., potential competition) or positive (e.g., potential

6 facilitation) associations between organisms remains a fundamental challenge of community

7 ecology (e.g., Barner, Coblentz, Hacker, \& Menge, 2018; Dormann et al., 2018; Harris, 2016;

8 Ovaskainen et al., 2017). Despite the challenges of detecting associations between co-

9 occurring species, associations between species are thought to be essential for structuring

10 free-living communities (e.g., de Araújo, Marcondes-Machado, \& Costa, 2014). For example,

11 associations between forest animal communities were more important in structuring

12 communities than habitat attributes such as vegetation (Le Borgne et al., 2018). In contrast,

13 the roles that interspecific associations play in governing the assembly of microbial systems

14 (microbial interspecific associations) are less understood (Ganz et al., 2017; Herren \&

15 McMahon, 2018; Zelezniak et al., 2015), particularly for within-host microbial communities.

16 Even when microbial interspecific associations are quantified in infra-communities, the

17 relative importance of microbe dispersal (Evans, Martiny, \& Allison, 2017; Zhou \& Ning,

18 2017) and host characteristics in shaping microbial infra-communities is rarely assessed

19 (Clark, Wells, \& Lindberg, 2018b). The quantification of interspecific associations of gut

20 microbes is rare in wildlife populations but, given the significant ecological insights derived

21 from studies of human gut microbial communities (e.g., Faust et al., 2012), is an important

22 knowledge gap to fill.

24 While our understanding of how microbial communities are structured is poor, it remains a

25 key goal in microbial ecology, particularly given microbial community relevance to animal 
26 health. Host characteristics such as sex, body condition, and the presence or absence of

27 pathogens are recognised as important for shaping microbial infra-communities (Britton \&

28 Young, 2014; Cheng et al., 2015; Ganz et al., 2017; Hooper, Littman, \& Macpherson, 2012;

29 Jani \& Briggs, 2014, 2018; McKenney et al., 2015; Mshelia et al., 2018; Näpflin \& Schmid-

30 Hempel, 2018). For example, horses in poor body condition have greater microbial diversity

31 and a different suite of microbial species present compared to horses in good body condition

32 (Mshelia et al., 2018). High loads of the pathogen Batrachochytrium dendrobatidis can not

33 only increase amphibian skin microbial diversity but also alter microbial composition (Jani \&

34 Briggs, 2014, 2018). However, the relative importance of host characteristics compared to

35 other ecological processes, such as interspecific associations, in structuring microbial infra-

36 communities is poorly understood.

38 Understanding how microbial infra-communities are shaped, however, is technically

39 challenging, in part due to the complexity of untangling complex ecological processes in

40 often species-rich but poorly characterized communities (Zhou \& Ning, 2017). Graphical

41 network models such as conditional random fields (CRF) offer exciting opportunities to

42 address this challenge by estimating associations between organisms in a community (Clark,

43 Wells, \& Lindberg, 2018b). In effect, these models can untangle relative influences of

44 microbial interspecific associations and host characteristics in predicting community

45 compositions. Crucially, inferences gleaned from CRFs can be integrated with phylogenetic

46 or functional data to provide new insights into mechanisms underlying microbial community

47 assembly. For example, associations between microbes may be non-random in that

48 phylogenetically or functionally similar species may co-occur more frequently (e.g., Bauer \&

49 Thiele, 2018). However, non-random associations detected in the human gut have been found

50 to be often negative and between phylogenetically or functionally distinct bacterial species, 
51 indicating that competition is likely an important driver of structure in this community (Faust

52 et al., 2012). Overlaying information such as this on graphical models has the potential to

53 highlight the broader mechanisms shaping microbial infra-communities.

55 Here we use a CRF approach to investigate microbial community composition in a wild

56 moose (Alces alces) population in Minnesota. Over the last two decades, moose in

57 Minnesota, which exist on the southern edge of their species range, have experienced

58 significant population decreases (Delgiudice, 2018; Lenarz, 2009). The most dramatic decline

59 has been reported for moose in northwest Minnesota, where the population declined from

604,000 animals in the 1980 s to less than 100 by the mid-2000's due to a combination of

61 pathogens and malnutrition (Murray et al., 2006). Recently, moose in northeast Minnesota

62 have experienced a 55\% population decline, driven largely by parasitic infections in adults

63 and wolf predation in calves (Carstensen et al., 2018; Severud, 2017; Wünschmann et al.,

64 2015). Moose gut microbial communities are known to vary with sex and age (Ishaq,

65 Sundset, Crouse, \& Wright, 2015; Ishaq \& Wright, 2012), but to what extent these host

66 characteristics, as well as pathogens and malnutrition, shape moose gut microbial

67 communities is unknown. Other ecological processes such interspecific assocations, even

68 though rarely quantified in wild populations, are also likely to play a role in shaping the

69 moose gut microbial community as they do for other ruminants (Henderson et al., 2015).

70 Furthermore, animals within close proximity to each other may have similar gut microbial

71 communities due to similarities in diet and/or increased possibilities for microbial dispersal

72 between individuals; both factors known to be important in gut microbial community

73 assembly in many host species (Henderson et al., 2015; Moeller et al., 2017). 
75 Here we apply a novel CRF approach that can quantify the relative importance of host

76 characteristics (including moose sex, body condition, pathogen exposure, and pregnancy

77 status) and microbial interspecific associations in shaping moose gut communities whilst

78 accounting for underlying spatial autocorrelation in microbial occurrences. Accounting for

79 spatial autocorrelation not only reduces false detection of interspecific associations that may

80 arise due to dispersal limitation or diet but quantifies how important these processes could be

81 in shaping microbial distributions. Specifically, we address the following questions:

82 1. Do CRF model combinations including host characteristics and spatial proximity outperform models reflecting just associations between microbes in explaining microbial communities?

2. After controlling for host characteristics and spatial patterns are there any remaining non-random negative or positive associations between microbes?

3. If there are non-random associations, are they between microbes that are functionally

89 We hypothesized that host characteristics would be the dominant processes shaping the

90 moose gut microbial community, and that spatial proximity between hosts and interspecific

91 associations would also partially explain gut microbial co-occurrence.

\section{Materials and Methods}

\section{Sample collection and sequencing}

96 Faecal samples were collected from 55 wild moose that were part of companion studies of

97 survival and cause-specific mortality led by the Minnesota Department of Natural Resources,

98 Grand Portage Band of Lake Superior Chippewa, and Voyageur's National Park across north-

99 eastern Minnesota (Fig. 1), placed on ice until transportation to the laboratory, and stored at -

$10020^{\circ} \mathrm{C}$ prior to processing. This included moose that were live-captured $(n=52,2011-2015)$ 
101 and sick ( $n=3,2009-2010)$. Details of the capture and handling of these moose can be found

102 in Butler et al. (2012) and Carstensen et al. (2018). The metadata provided from these moose

103 included date and location of capture, pregnancy status, sex, age, body condition, and

104 serological exposure to Borrelia burgdorferi, West Nile Virus, and leptospira (6 serovars

105 including L. bratislava, L. canicola, L. grippotyphosa, L. hardjo, L. icterohemorrhagica and

106 L. pomona). In total, we had serological data for 49 moose (33 females and 16 males) with

107 the remaining 6 individuals removed from the CRF analysis (but retained for the descriptive

108 analysis). See Butler et al. (2012) for serological test details. Of the live-captured moose, 21

109 were observed to be underweight (thin/very thin), and 31 were considered of normal body

110 condition at winter capture (Jan-Feb). All samples apart from one were collected in winter

111 between 2011-2015; the other sample was collected in 2003. Microbial DNA was extracted

112 from the faecal samples using the PowerSoil DNA Isolation kit (Qiagen), in accordance with

113 the manufacturer's protocol. The V4 hypervariable region of the bacterial 16S rRNA gene

114 was amplified using the barcoding primers 515F and 806R. Amplicons were sequenced on

115 the Illumina MiSeq platform following the method outlined by Gohl et al., (2016).

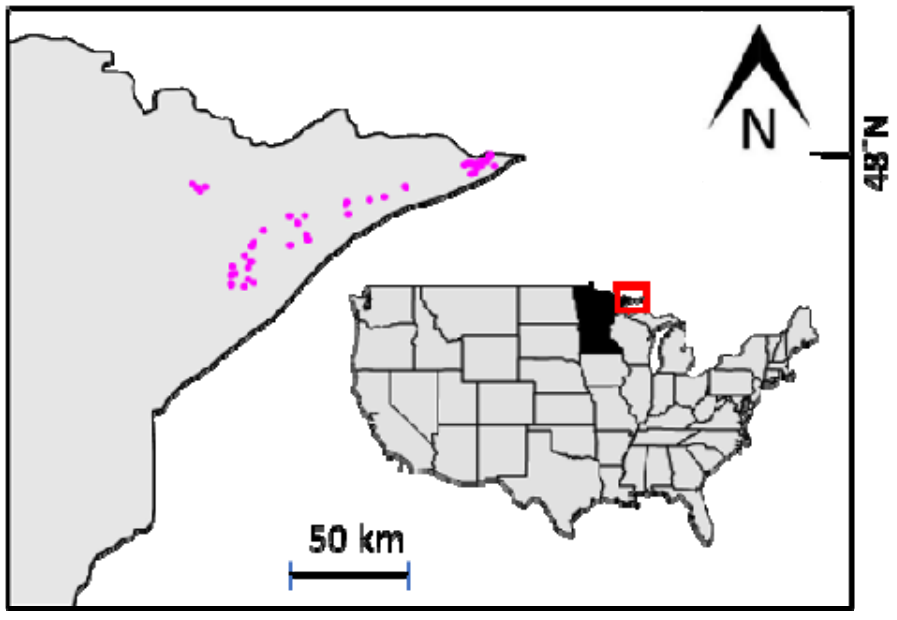

117 Figure 1: Locations (pink dots) of moose faecal samples in northeastern Minnesota, USA.

118 The red box shows the location of the study in Minnesota. 
120

121

122

123

124

125

126

127

128

129

130

131

132

133

134

135

136

137

138

\section{Bioinformatics pipeline}

Raw sequencing reads were processed using the University of Minnesota's metagenomics-

pipeline (complete description of the pipeline can be found at

https://bitbucket.org/jgarbe/gopher-pipelines/overview), which implements the QIIME

version 1.9.1 analysis software (Caporaso et al., 2010). Briefly, sequencing adaptors were

trimmed using Trimmomatic (Bolger, Lohse, \& Usadel, 2014) and read pairs were assembled

and primer sequences removed using PANDAseq (Masella, Bartram, Truszkowski, Brown, \&

Neufeld, 2012). Reads without primers, unpaired reads, and assembled reads that were

outside the expected rRNA gene V4 region length were discarded. Chimeras were detected

and removed with QIIME's identify_chimeric_seqs.py function with the usearch61 algorithm

(Edgar, 2010). Open-reference OTU (operational taxonomic unit) picking was conducted

using QIIME's pick_open_reference_otus.py with a minimum sequence identity threshold of 97\%. Representative OTU sequences were aligned against the Greengenes 18.8 core set

(DeSantis et al., 2006b) using UCLUST (Edgar, 2010) with QIIME default parameters.

Singleton OTUs and those that did not align with PyNAST (Caporaso et al., 2010) were

removed from the analysis. To control for differences in sequencing depth between samples,

read counts were rarefied to the lowest number of reads $(101,131)$ per sample.

To test for co-occurrence patterns between gut microbes, we filtered out OTUs with $\leq 10 \%$

abundance (i.e., only keeping OTUs that occurred in at least $10 \%$ of samples) and converted

the OTU table into a presence-absence matrix. OTUs that were found $\geq 75 \%$ of samples were

also filtered from the analysis. Our purpose for removing rare and common OTUs was to

ensure that adequate inferences could be made about the occurrence probability of each OTU

(Ovaskainen, Abrego, Halme, \& Dunson, 2016). This would be difficult / impossible to do if the target OTU shows little variability across sampled environmental gradients by being either too rare or too common. We transformed the presence-absence matrix into a Jaccard 
147 similarity matrix and used non-metric multidimensional scaling (NMDS) to select OTUs that

148 were driving compositional change across the moose samples and therefore likely to differ

149 with moose characteristics such as body condition (Pearson correlation coefficient $>0.45$ ). In

150 total, 42 OTUs remained after this filtering process. NMDS was performed using functions in

151 the R package vegan (Oksanen et al., 2013).

153 To reduce the dimensions of the serological exposure data for subsequent analysis, we

154 converted test results into a binary matrix ( 0 if the individual tested negative and 1 if

155 positive), transformed the binary matrix into a Jaccard similarity matrix, and then performed

156 principal coordinate analysis (PCoA) using the R package vegan (Oksanen et al., 2013). The

157 first three PCoA axes represented $85 \%$ of the variation in exposure across individuals and

158 eigenvalues from these axes were used as covariates in the CRF analysis below (now called

159 pathogen exposure PCoA axes).

\section{Conditional random fields}

\section{Identifying OTU co-occurrence patterns using conditional random fields}

164 The framework we used to investigate OTU co-occurrence probabilities while accounting for 165 potential influences of covariates is described in detail by Clark et al. (2018a) and references 166 therein. Briefly, the log-odds of observing OTU $j$ given covariate $x$ and the presence-absence 167 of OTU $k$ is modelled using:

$$
\log \left(\frac{P\left(y_{j}=1 \mid y_{\backslash j}, x\right)}{1-P\left(y_{j}=1 \mid y_{\backslash j}, x\right)}\right)=\alpha_{j 0}+\beta_{j}^{T} x+\sum_{k: k \neq j}\left(\alpha_{j k 0}+\beta_{j k}^{T} x\right) y_{k}
$$

168 where $y_{j}$ is a vector of binary observations for OTU $j$ ( 1 if the OTU was present, 0 if absent),

$169 y_{\mathrm{j}}$ represents vectors of binary observations for all other OTUs apart from $j, \alpha_{j 0}$ is the OTU-

170 level intercept, and $\beta_{j}^{T}$ is the coefficient for the effect of covariate $x$ on OTU $j$ 's occurrence 
171 probability. Interaction parameters are represented by $\alpha_{j k 0}$ and $\beta_{j k}^{T} x$ (defined below).

172 Parameterization of the likelihood is estimated using logistic regression, where regression

173 coefficients represent the effects of predictors on the OTU's conditional log-odds. Cross-

174 multiplying all combinations of co-occurring OTUs and external covariates allows direct

175 comparison of the relative influences interspecific associations) and host effects on an OTU's

176 occurrence probability. For each OTU-specific regression, sparsity is added to the model

177 using L1 (e.g., least absolute shrinkage and selection operator [LASSO]) regularisation to

178 force regression coefficients toward zero if they have minimal effects. Ten-fold cross-

179 validation was implemented to choose the penalty that minimised cross-validated error, as

180 this is considered an appropriate loss function in binomial classification studies. Following

181 LASSO variable selection, coefficients representing conditional dependence of two OTUs

182 and coefficients representing effects of covariates on this dependence were symmetrized by

183 taking the mean of the corresponding estimates so that $\alpha_{j k 0}=\alpha_{k j 0}$ and $\beta^{T}{ }_{j k} x=\beta^{T}{ }_{k j} x$. This means

184 we can approximate parameters from a unified graphical network, after maximizing the

185 conditional log-likelihood for each OTU (Lee \& Hastie, 2015). Following unification,

186 inference is straightforward. If $\alpha_{j k 0}=0$, we can infer that the occurrence probabilities of

187 OTUs $j$ and $k$ are conditionally independent, after accounting for covariates and other OTUs.

188 If $\alpha_{j k 0} \neq 0$ but $\beta_{j k}^{T} x=0$, the occurrence probabilities are still considered conditionally

189 dependent, but the strength of this dependence is not expected to co-vary with covariate $x$.

MRF and CRF model selection

192 We estimated four graphical model formulations of increasing complexity to identify a best-

193 fitting model for our OTU presence-absence dataset. In the first, we built a Markov random

194 fields (MRF) graph that did not include any spatial data or host characteristics and used only

195 the binary occurrences of the 42 OTUs as predictors (hereafter the MRF model). In the 
196 second model, the GPS coordinates for each observation (latitude and longitude, in decimal

197 degrees) were used to construct penalised Gaussian process regression splines with 100

198 degrees of freedom (Kammann \& Wand, 2003; Wood, 2003) (hereafter the spatial MRF

199 model). Including the spatial splines in each OTU's linear predictor ensured that interspecific

200 associations on occurrence probabilities were estimated only after accounting for possible

201 spatial autocorrelation. For the third model, we built a CRF including moose characteristics

202 covariates (moose sex, body condition, pregnancy status and the pathogen exposure PCoA

203 axes) as well as longitude and latitude (hereafter the non-spatial CRF model). All covariates

204 were included as scaled continuous variables with the exceptions of sex and pregnancy status,

205 which were both included as categorical variables. For the final model, we built a CRF as

206 above, but we replaced the latitude and longitude variables with spatial splines (hereafter the

207 spatial CRF model).

209 We assessed the fit of each of the above candidate models to the observed data by calculating

210 the proportion of observations that each model successfully classified. This was done using

211 ten-fold cross-validation. The best-fitting model was then fit to 100 bootstrapped versions of

212 the observed data (randomly shuffling observations in each bootstrap iteration) to capture

213 uncertainty in model parameters. All CRF model fitting was performed using functions in the

214 MRFcov R package (Clark, Wells, \& Lindberg, 2018a). From the OTU co-occurrence data,

215 we constructed an adjacency matrix and plotted association networks using iGraph $\mathrm{R}$

216 package (Csárdi \& Nepusz, 2006). See Appendix S1 for R code detailing data preparation,

217 analytical routine and model specification. 
221 We predicted the molecular functions of OTUs using PICRUSt's precalculated functional

222 prediction table, where the rows were KEGG orthologs (KOs) and the columns were OTUs

223 based on Greengeens identification numbers (DeSantis et al., 2006a; Kanehisa, Sato,

224 Kawashima, Furumichi, \& Tanabe, 2016; Langille et al., 2013). This KO table was converted

225 into a presence-absence matrix (i.e., whether a particular functional ortholog is associated

226 with that OTU) and calculated the pairwise functional similarity using the Jaccard index. We

227 applied NMDS to view the broad functional relationships between OTUs and define

228 functional groups (FGs).

\section{Results}

230

231

We found that Firmicutes, followed by Bacteroidetes, were the dominant phyla in all our

moose gut communities making up over $75 \%$ of the reads detected (Fig. S1). The ratio of

these groups across samples was relatively consistent with only one individual having a

234 proportion $<50 \%$ of Firmicutes present (Fig. S2). Functionally the OTUs could be grouped

235 into two FGs (Fig. S3).

\section{Model fit}

238

The MRF model, which only quantified associations between microbes, could more

240 accurately predict OTU occurrence compared to either the spatial CRF or non-spatial CRF

241 models (both of which included host characteristics; Fig. 2a). Including spatial regression

242 splines did improve the fit of the CRF model, with this model accurately predicting $78 \%$ of

243 observations compared to $74 \%$ of for the non-spatial CRF (Fig. 2a). In contrast, the MRF

244 could accurately predict $83 \%$ of presence-absence observations on average (Fig. 2a). This

245 result was not due to one model being better able to predict occurrence over absence, as the

246 MRF had much higher specificity and sensitivity than either of the CRF models (Fig. 2b/c).

247 However, we still found evidence of spatial clustering in microbial occurrence probabilities, 
248 as MRF performance improved further when we included spatial splines in the model (Fig.

249 2a). Adding spatial data to this model enabled us to correctly predict $~ 90 \%$ of OTU

250 occurrences in the moose gut microbial community. This not only suggests that microbes in

251 our dataset were more likely to show similar occurrences in moose that were sampled nearby

252 to one another, but that false associations between microbes could be inferred by models that

253 do not account for spatial proximity.
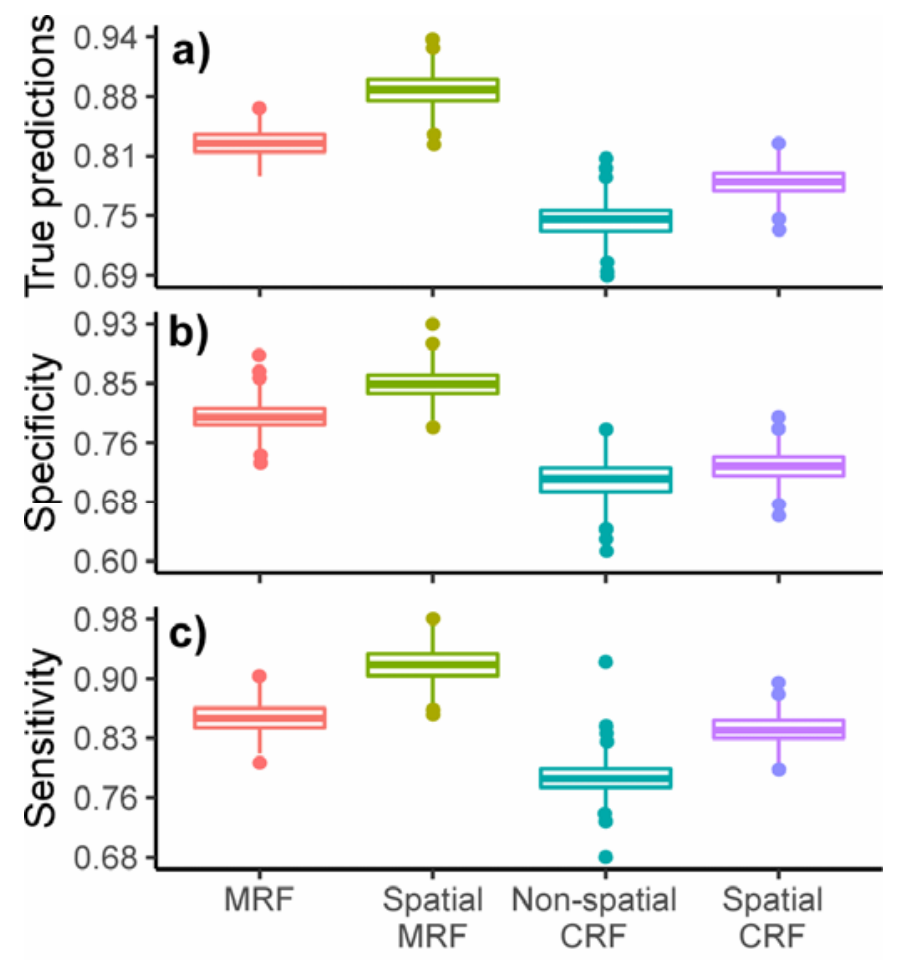
Model

255 Figure 2: Box and whisker plots showing the predictive performance of the Markov random

256 field model (MRF - without model covariates), spatial MRF model, non-spatial conditional

257 random field model (CRF - with model covariates) and spatial CRF as defined by (a) true

258 prediction performance, b) specificity, and c) sensitivity. Predictions were assessed by fitting

259 models to a random fold containing $90 \%$ of the data (training data) and predicting

260 observations for the remaining $10 \%$ (test data). This process was repeated 100 times to

261 capture uncertainty in performance. Specificity is the ability of the model to correctly identify

262 individual moose without the specified OTU (proportion of observed negatives that were 
263 predicted to be negative), while sensitivity is the ability to correctly identify individuals with

264 the specified OTU (proportion of observed positives that were predicted to be positive). Box

265 hinges show the interquartile range (25\% and $75 \%$ quantiles), lines within boxes indicate the

266 median (50\% quantile), whiskers show $10 \%$ and $90 \%$ quantiles and dots show values outside

267 these quantiles.

\section{Microbial associations}

270

271

The co-occurrence network revealed that both positive and negative associations occurred

272 across taxonomic and functional groups with no clear phylogenetic pattern (i.e., OTUs from

273 the same phyla/class or function were not preferentially negatively or positively associated

274 with each other, Fig. 3). On average, we found that positive associations between OTUs were

275 more common than negative associations (4 vs 2, Table 1). Firmicutes was the best-

276 represented phyla in our moose faecal samples (Fig. S1), and this phylum also had the highest

277 number of OTU associations. Strikingly, even though Bacteroidetes was the second most

278 dominant phyla, we did not detect any associations involving OTUs from this taxa. In

279 contrast, OTUs from Tenericutes were rare (3\% of sequences, Fig. S1), but overall, they had

280 on average 1 more positive association partner than other phyla (4 vs 3$)$, whereas

281 Cyanobacteria OTUs had more negative association partners than other phyla (3 vs 2, Table

282 1). FGs showed similar differences, with FG1 (which was dominated by the Tenericutes; Fig.

283 S3) having more positive associations compared to FG2 (4 vs 3 on average). The opposite

284 was true for negative associations, with FG2 having more associations on average than FG1

285 (1 vs 2). Overall, two previously uncharacterized OTUs (identified by the 'NewReference'

286 label) from class Mollicutes (Tenericutes) and class Clostridia (Firmicutes) had the highest

287 number of associations overall (8, Table 1). Two OTUs from class Clostridia had the highest

288 numbers of negative associations ( $5 \& 6$ respectively, Table 1 ). 
bioRxiv preprint doi: https://doi.org/10.1101/514604; this version posted January 9, 2019. The copyright holder for this preprint (which was not certified by peer review) is the author/funder, who has granted bioRxiv a license to display the preprint in perpetuity. It is made available under aCC-BY-NC-ND 4.0 International license.

289 Table 1: Summary of associations detected in the MRF analysis.

\begin{tabular}{|c|c|c|c|c|c|}
\hline OTU identity & Avg.oc & Pos & Neg & Phylum|Class & FG \\
\hline 338145 & 0.2043 & 6 & 4 & Actinobacteria|Coriobacteriia & 2 \\
\hline NewReferenceOTU314 & 0.6637 & 2 & 1 & Actinobacteria|Coriobacteriia & $\mathrm{N}$ \\
\hline NewReferenceOTU69 & 0.6832 & 2 & 1 & Actinobacteria|Coriobacteriia & $\mathrm{N}$ \\
\hline NewReferenceOTU74 & 0.7562 & 3 & 2 & Actinobacteria|Coriobacteriia & $\mathrm{N}$ \\
\hline 206930 & 0.4799 & 4 & 4 & Cyanobacteria|4COd-2 & 2 \\
\hline NewReferenceOTU123 & 0.5473 & 6 & 3 & Cyanobacteria $4 \mathrm{C} 0 \mathrm{~d}-2$ & $\mathrm{~N}$ \\
\hline NewReferenceOTU73 & 0.5461 & 0 & 2 & Cyanobacteria|Chloroplast & $\mathrm{N}$ \\
\hline 322906 & 0.9437 & 3 & 4 & Firmicutes|Clostridia & 2 \\
\hline 325706 & 0.6148 & 0 & 1 & Firmicutes|Clostridia & 2 \\
\hline 4317006 & 0.0372 & 5 & 4 & Firmicutes|Clostridia & 2 \\
\hline 4480841 & 0.5291 & 2 & 1 & Firmicutes|Clostridia & 2 \\
\hline 4314603 & 0.4536 & 2 & 1 & Firmicutes|Clostridia & 2 \\
\hline 4417708 & 0.0843 & 7 & 4 & Firmicutes|Clostridia & 2 \\
\hline 296918 & 0.7692 & 1 & 4 & Firmicutes|Clostridia & 2 \\
\hline 266952 & 0.6309 & 3 & 3 & Firmicutes|Clostridia & 2 \\
\hline 294064 & 0.5084 & 5 & 3 & Firmicutes|Clostridia & 2 \\
\hline 294262 & 0.2403 & 6 & 1 & Firmicutes|Clostridia & 2 \\
\hline 579159 & 0.2068 & 3 & 2 & Firmicutes|Clostridia & 2 \\
\hline 333577 & 0.3276 & 2 & 1 & Firmicutes|Clostridia & 2 \\
\hline 1038874 & 0.3899 & 2 & 0 & Firmicutes|Clostridia & 2 \\
\hline 129755 & 0.3879 & 3 & 0 & Firmicutes|Clostridia & 2 \\
\hline 738351 & 0.6364 & 1 & 0 & Firmicutes|Clostridia & 2 \\
\hline 4295783 & 0.3693 & 1 & 0 & Firmicutes|Clostridia & 2 \\
\hline 574585 & 0.6326 & 1 & 5 & Firmicutes|Clostridia & 2 \\
\hline NewReferenceOTU108 & 0.0809 & 8 & 3 & Firmicutes|Clostridia & $\mathrm{N}$ \\
\hline NewReferenceOTU74 & 0.3172 & 1 & 0 & Firmicutes|Clostridia & $\mathrm{N}$ \\
\hline NewReferenceOTU76 & 0.2891 & 5 & 6 & Firmicutes|Clostridia & $\mathrm{N}$ \\
\hline NewReferenceOTU76 & 0.0827 & 6 & 4 & Firmicutes|Clostridia & $\mathrm{N}$ \\
\hline NewReferenceOTU157 & 0.0899 & 3 & 0 & Firmicutes|Clostridia & $\mathrm{N}$ \\
\hline NewReferenceOTU289 & 0.1515 & 5 & 4 & Firmicutes|Clostridia & $\mathrm{N}$ \\
\hline 4396877 & 0.0928 & 5 & 1 & Firmicutes|Erysipelotrichi & 2 \\
\hline NewReferenceOTU42 & 0.5286 & 3 & 4 & Firmicutes|Erysipelotrichi & $\mathrm{N}$ \\
\hline 339838 & 0.063 & 1 & 1 & Tenericutes|Mollicutes & 1 \\
\hline 513605 & 0.0299 & 7 & 1 & Tenericutes|Mollicutes & 1 \\
\hline 4446732 & 0.6627 & 4 & 2 & Tenericutes|Mollicutes & 1 \\
\hline 1108356 & 0.5123 & 4 & 2 & Tenericutes|Mollicutes & 1 \\
\hline 921020 & 0.1596 & 5 & 1 & Tenericutes|Mollicutes & 1 \\
\hline NewReferenceOTU413 & 0.2271 & 5 & 2 & Tenericutes|Mollicutes & $\mathrm{N}$ \\
\hline NewReferenceOTU493 & 0.2445 & 4 & 1 & Tenericutes|Mollicutes & $\mathrm{N}$ \\
\hline NewReferenceOTU390 & 0.6923 & 0 & 0 & Tenericutes|Mollicutes & $\mathrm{N}$ \\
\hline NewReferenceOTU404 & 0.1345 & 8 & 2 & Tenericutes|Mollicutes & $\mathrm{N}$ \\
\hline NewReferenceOTU528 & 0.4842 & 4 & 4 & Tenericutes|RF3 & $\mathrm{N}$ \\
\hline Average & & 4 & 2 & & \\
\hline
\end{tabular}

290 Avg.oc: average occurrence in our moose samples. Pos: number of positive associations.

291 Neg: number of negative associations. 'NewReference' indicates that the OTU has not been

292 previously characterised by the Greengenes database. FG: Functional group (see Figure S3).

293 N: No functional group could be determined (not previously characterized). 


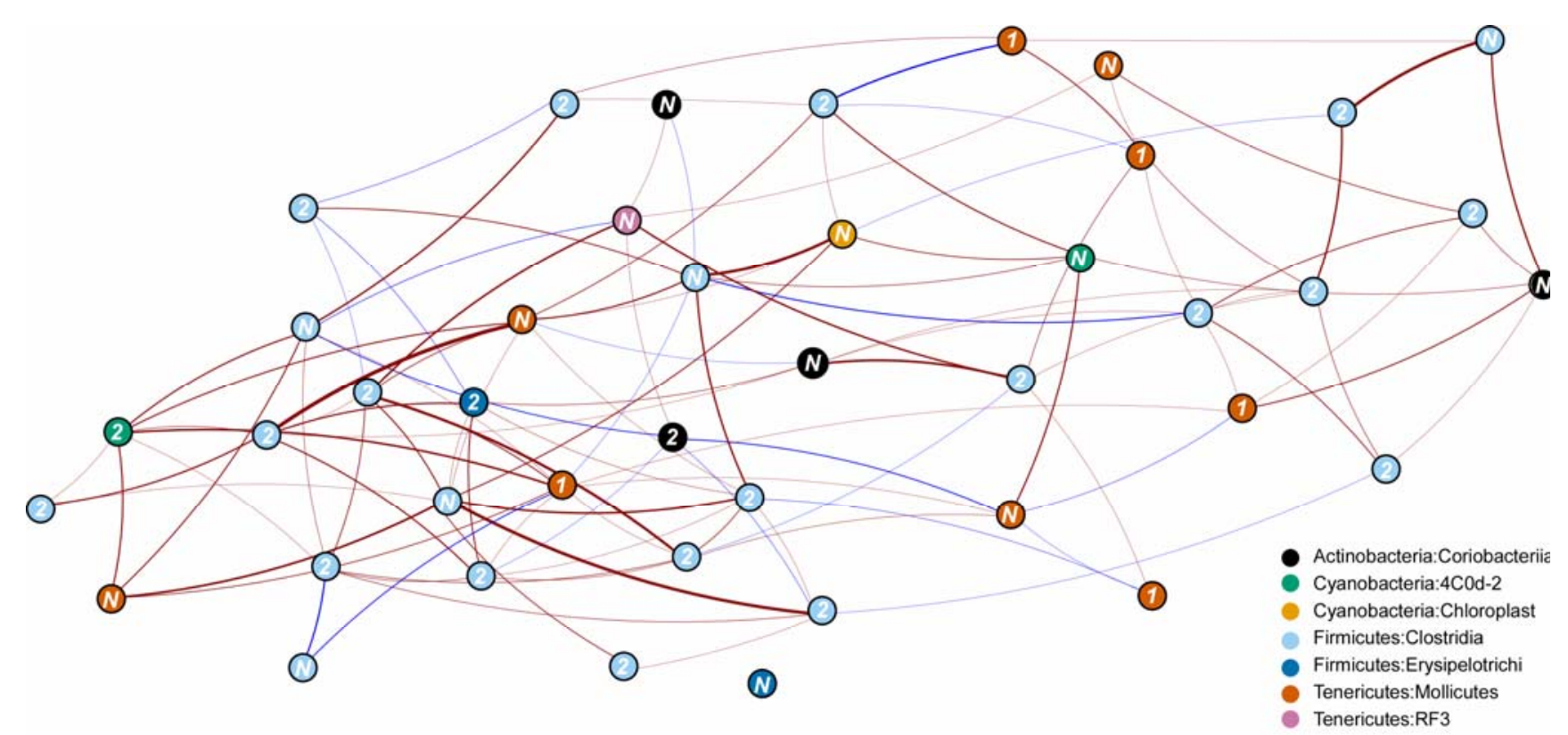

296 Figure 3: Moose gut microbial MRF co-occurrence network. Blue edges indicate negative

associations and red edges represent positive associations. Edge thickness is scaled by the

strength of association. Node colour indicates taxonomic group of each microbe. Numbers on

the node represent which broad functional group (FG) each OTU belonged to (see Fig. S3).

300

'N' indicates that there was no functional data for this microbe. See Fig. S4 for the OTU

301

correlation matrix with names included.

\section{Discussion}

Utilizing graphical network models, we show the importance of microbial interspecific

associations over host characteristics in shaping moose gut microbial communities at a

population scale. In this case, both interspecific associations and spatial proximity were

important for shaping microbial communities in this declining moose population. Host 
312 and positive microbial associations with no clear functional or phylogenetic pattern. Our

313 study not only highlights the importance of accounting for interspecific associations when

314 trying to quantify how host characteristics shapes host infra-communities but also shows the

315 value of graphical network models in untangling community dynamics more broadly.

317 We found that evidence of pathogen exposure was not particularly important in predicting

318 moose gut microbial community dynamics. As the serological evidence we used in this study

319 only infers past infection by a particular pathogen rather than current infection status, perhaps

320 this is not surprising. Previous studies testing the role of pathogens shaping microbial

321 communities have used evidence of current infection (e.g., qPCR; Ganz et al., 2017;

322 McKenney et al., 2015) rather than previous exposure based on serological evidence. When

323 we sampled the moose gut microbial communities, the animal may not be experiencing the

324 infection, and this may be the reason we did not detect an effect. It is also possible that

325 herbivore gut microbial communities are more resilient to pathogen infection than other

326 trophic groups such as carnivores. Ruminant microbial communities are more dominated by

327 environmental bacteria cultivated by the host to aid digestion (Muegge et al., 2011) and may

328 be less regulated by the immune system compared to the gut microbial communities in other

329 species (Ley et al., 2008). Lower immune regulation, for example, could explain why the

330 herbivores such as the gorilla (Gorilla gorilla) have microbiomes remarkably resilient to

331 Simian immunodeficiency virus infection compared to omnivorous chimpanzees (Pan

332 troglodytes) or for HIV infection in humans (Moeller et al., 2015). In support of this, we find

333 that across individual moose in our study, the relative proportions of gut microbial taxa were

334 relatively consistent, despite large differences in body condition and exposure status (Fig.

335 S2). Similar ratios of the different microbial taxa in moose have also been reported in moose

336 from the east coast of North America (Ishaq \& Wright, 2012) even though samples were 
337 collected from moose in a different season. Further studies where current pathogen infection

338 status is known in moose and other herbivores are needed to resolve this question.

340 Interspecific associations, in contrast, were much better predictors of moose gut microbial

341 communities. In free-living communities, the role of interspecific associations in shaping the

342 distributions of species is increasingly recognised (e.g., Aragón, Carrascal, \& Palomino,

343 2018; C. B. de Araújo et al., 2014). However, the importance of interspecific associations is

344 likely to decrease with geographic scale (Araújo \& Rozenfeld, 2014). Whether this applies to

345 gut microbial communities, in contrast, is poorly studied. In our case, host effects may have

346 been detectable if we sampled a greater number of individuals across a larger portion of

347 moose home range in central North America. Nonetheless, the scale at which wildlife

348 surveillance is performed (and thus faecal samples collected for gut microbiome analysis) is

349 often similar across studies (e.g., Cheng et al., 2015). In general, understanding the role that

350 scale plays in structuring gut microbial communities, beyond the population scale, is a major

351 challenge for microbial ecology (Antwis et al., 2017; Camp, Kanther, Semova, \& Rawls,

352 2009). Based on our findings, we suggest that larger geographical and/or temporal scales are

353 needed to detect possible impacts of host characteristics on the structure of gut microbial

354 communities.

356 Even without accounting for host traits such as host genetics that are thought to be important

357 in shaping the gut microbial communities in other herbivores (Kohl, Varner, Wilkening, \&

358 Dearing, 2017), our models had high predictive performance. Our results may be due to

359 samples coming from a relatively well-mixed population, even though our moose samples

360 were taken over a large area spanning a maximum of $\sim 150 \mathrm{~km}$. Central North America

361 (including Minnesota) has the highest genetic diversity of all North American moose 
362 populations (Hundertmark, Bowyer, Shields, \& Schwartz, 2003), but to what extent our

363 samples come from genetically distinct populations is unknown. Future work incorporating

364 host genetics in moose may help better understand the role of host characteristics, compared

365 to microbial interspecific associations in shaping infra-communities.

367 Our capacity to predict the moose gut microbial community at the population scale increased

368 when we included spatial information, indicating that either stochastic events such as

369 microbial dispersal or spatial similarities in diet may be important in structuring associations

370 between gut microbes. Dispersal of microbes is thought to be passive (Nemergut et al., 2013),

371 however, these microbial species are often in high abundance and have broad distributions

372 making dispersal challenging to quantify (Evans et al., 2017; Zhou \& Ning, 2017).

373 Biogeographical studies have shown that dispersal limitation is important for structuring

374 microbial communities including those of the gut (Evans et al., 2017; Hanson, Fuhrman,

375 Horner-Devine, \& Martiny, 2012; Moeller et al., 2017), but rarely over a relatively small area

376 within a host population. What role moose diet explicitly played in shaping our moose gut

377 microbial communities is still an open question. Future studies linking host microbial data to

378 measures of host diet, such as stable isotope analysis (Hofman-Kamińska, Bocherens,

379 Borowik, Drucker, \& Kowalczyk, 2018), will enable dispersal limitation and diet to be

380 decoupled in future models. Nonetheless, we show that including host spatial data in future

381 work is necessary for robustly quantifying microbial co-occurrence patterns.

383 We also found strong positive and negative associations between OTUs from different phyla

384 and divergent functional groups in the moose gut microbial community. Clostridia

385 (Firmicutes) and Mollicutes (Tenericutes) were network 'hubs' involved in a relatively high

386 number of positive associations in our moose population, and often with each other (Table 1). 
387 Clostridia OTUs are also hubs for associations in the human gut community (Banerjee,

388 Schlaeppi, \& van der Heijden, 2018; Faust et al., 2012), but the importance of Mollicutes has

389 not been, to our knowledge, reported elsewhere. Moreover, we detected no negative

390 associations between Bacteroidetes and Firmicutes even though Bacteroidetes was common

391 in our samples; this group may not play a dominant role in structuring moose gut microbial

392 communities as they do in humans (Banerjee et al., 2018; Faust et al., 2012). Whether the

393 same relationships apply in herbivore populations where Bacteroidetes is dominant, as was

394 the case in an Alaskan and Swedish moose (Ishaq \& Wright, 2014; Svartström et al., 2017),

395 remains an open question. More generally, Faust et al. (2012) found an increased likelihood

396 of negative associations between OTUs that were functionally and phylogenetically

397 dissimilar (with opposite true for more related OTUs). We did not see such a pattern in our

398 co-occurrence network and why we would get such a different result in moose is unclear. The

399 Faust dataset consisted of 240 individuals from the Human Microbiome Project (Methé et al.,

400 2012), but large methodological differences make direct comparison difficult, as factors such

401 as spatial relationships between subjects were not quantified. How robust these relationships

402 are also likely to be impacted by taxonomic resolution of each network (Faust et al., 2012).

403 As we used 16S rRNA gene sequencing, we were unable to get sequence identifications to

404 species level. Further, functional profiles were based on predictions from reference genomes,

405 not direct identification of functional genes or proteins (Langille et al., 2013). Shotgun

406 metagenomic sequencing could allow for both classification sequences to species-level and

407 provide much more detailed insights into the functional patterns shaping microbial species

408 associations.

409

410 Here we have demonstrated that graphic network models can untangle how interspecific

411 interactions can shape gut microbial communities in moose. Additionally, we show that MRF 
412 and CRF models can robustly construct co-occurrence networks (Clark, Wells, \& Lindberg,

413 2018b) and, coupled with taxonomic and functional information, find high-resolution insights

414 into interspecific associations. Graphical network models, as with other correlation-based

415 approaches, do have limitations. Correlations identified by techniques such as MRF or CRF

416 should be treated with caution as they do not imply causation (e.g., Barner et al., 2018;

417 Dormann et al., 2018). Follow up analysis with tools such as structural equation modelling

418 (SEM) could be used to go beyond correlations to explore potential causal relationships

419 between microbes (Banerjee et al., 2018). Nonetheless, by explicitly incorporating spatial

420 data and covariates into graphical models, our method offers a step forward in characterising

421 associations between species, and we envisage this method will be broadly useful for

422 researchers working on micro- and macro-community dynamics alike. Studies analysing

423 associations between infra-community microbial species are rare in wildlife, even though

424 they can provide important insights into the ecological dynamics operating within or on the

425 host. Given the decreasing cost of microbial surveys and analytical advances such as ours,

426 studies that can disentangle microbial infra-community dynamics in wildlife species will

427 become more frequent, and this can ultimately provide a more nuanced understanding of

428 wildlife health.

430 Acknowledgements

431 This material is based upon work supported by the Cooperative State Research Service, U.S.

432 Department of Agriculture, under Project No. MINV-62-051.

\section{Data accessibility}

436 All data will be made accessible in Dryad. 


\section{Author contribution statement.}

439

$440 \mathrm{NFJ}, \mathrm{MC}, \mathrm{TW}, \mathrm{JF}, \mathrm{TJ}, \mathrm{AK}$ and MEC came up with the project design. NFJ, NC and EM

441 conducted the analysis. NC coded the spatial MRF/CRF functions. MC, TW, SM and JF

442 provided data. All authors contributed critically to the drafts and gave final approval for

443 publication.

444

445

446

447

\section{References}

Antwis, R. E., Griffiths, S. M., Harrison, X. A., Aranega-Bou, P., Arce, A., Bettridge, A. S., ... Sutherland, W. J. (2017). Fifty important research questions in microbial ecology. FEMS Microbiology Ecology, 93(5). doi:10.1093/femsec/fix044

Aragón, P., Carrascal, L. M., \& Palomino, D. (2018). Macro-spatial structure of biotic interactions in the distribution of a raptor species. Journal of Biogeography, 45(8),

Araújo, M. B., \& Rozenfeld, A. (2014). The geographic scaling of biotic interactions.

Banerjee, S., Schlaeppi, K., \& van der Heijden, M. G. A. (2018). Keystone taxa as drivers of microbiome structure and functioning. Nature Reviews Microbiology, 16(9), 567-576. doi:10.1038/s41579-018-0024-1 contradictions among observational and experimental estimates of non-trophic species human gut microbiota. MSystems, 3(3), e00209-17. doi:10.1128/mSystems.00209-17

463 Bolger, A. M., Lohse, M., \& Usadel, B. (2014). Trimmomatic: a flexible trimmer for Illumina 
sequence data. Bioinformatics, 30(15), 2114-2120. doi:10.1093/bioinformatics/btu170

465 Britton, R. A., \& Young, V. B. (2014). Role of the intestinal microbiota in resistance to

466 colonization by Clostridium difficile. Gastroenterology, 146(6), 1547-1553.

467 doi:10.1053/j.gastro.2014.01.059

468 Butler, E., Carstensen, M., Hildebrand, E., \& Giudice, J. (2012). Northeast Minnesota moose

469 herd health assessment 2007-2012. In L. Cornicelli, M. Carstensen, M. Grund, M.

470 Larson, \& Lawrence JS (Eds.), Summaries of wildlife research findings 2012. St Paul:

471 Minnesota Department of Natural Resources. Retrieved from http://www.mndnr.gov

472 Camp, J. G., Kanther, M., Semova, I., \& Rawls, J. F. (2009). Patterns and scales in

473 gastrointestinal microbial ecology. Gastroenterology, 136(6), 1989-2002.

$474 \quad$ doi:10.1053/j.gastro.2009.02.075

475 Caporaso, J. G., Bittinger, K., Bushman, F. D., DeSantis, T. Z., Andersen, G. L., \& Knight,

476 R. (2010). PyNAST: a flexible tool for aligning sequences to a template alignment.

477 Bioinformatics, 26(2), 266-267. doi:10.1093/bioinformatics/btp636

478 Caporaso, J. G., Kuczynski, J., Stombaugh, J., Bittinger, K., Bushman, F. D., Costello, E. K., 479 ... Knight, R. (2010). QIIME allows analysis of high-throughput community sequencing 480 data. Nature Methods, 7(5), 335-336. doi:10.1038/nmeth.f.303

481 Carstensen, M., Hildebrand, E. C., Plattner, D., Dexter, M., St-Louis, V., Jennelle, C., \&

482 Wright, R. G. (2018). Determining cause specific mortality of adult moose in Northeast

483 Minnesota, February 2013 - July 2017. Retrieved from

484 https://files.dnr.state.mn.us/wildlife/research/summaries/health/2016_moose-

$485 \quad$ mortality.pdf

486 Chase, J. M., \& Myers, J. A. (2011). Disentangling the importance of ecological niches from 487 stochastic processes across scales. Philosophical Transactions of the Royal Society B:

488 Biological Sciences, 366(1576), 2351-2363. doi:10.1098/rstb.2011.0063 
489 Cheng, Y., Fox, S., Pemberton, D., Hogg, C., Papenfuss, A. T., \& Belov, K. (2015). The

490 Tasmanian devil microbiome-implications for conservation and management.

491 Microbiome, 3(1), 76. doi:10.1186/s40168-015-0143-0

492 Clark, N. J., Wells, K., \& Lindberg, O. (2018). MRFcov: Markov Random Fields with

493 additional covariates. R package version 1.0.33. Availabe at GitHub.

494 https://github.com/nicholasjclark/MRFcov.

495 Clark, N. J., Wells, K., \& Lindberg, O. (2018). Unravelling changing interspecific

496 interactions across environmental gradients using Markov random fields. Ecology,

497 99(6), 1277-1283. doi:10.1002/ecy.2221

498 Csárdi, G., \& Nepusz, T. (2006). The igraph software package for complex network research.

$499 \quad$ InterJournal Complex Systems, 1695.

500 de Araújo, C. B., Marcondes-Machado, L. O., \& Costa, G. C. (2014). The importance of

501 biotic interactions in species distribution models: a test of the Eltonian noise hypothesis

502 using parrots. Journal of Biogeography, 41(3), 513-523. doi:10.1111/jbi.12234

503 Delgiudice, G. D. (2018). 2018 Aerial Moose Survey. Retrieved from

504 https://files.dnr.state.mn.us/wildlife/moose/moosesurvey.pdf

505 DeSantis, T. Z., Hugenholtz, P., Larsen, N., Rojas, M., Brodie, E. L., Keller, K., ...

506 Andersen, G. L. (2006a). Greengenes, a chimera-checked 16S rRNA gene database and

507 workbench compatible with ARB. Applied and Environmental Microbiology, 72(7),

508 5069-72. doi:10.1128/AEM.03006-05

509 DeSantis, T. Z., Hugenholtz, P., Larsen, N., Rojas, M., Brodie, E. L., Keller, K., ...

510 Andersen, G. L. (2006b). Greengenes, a chimera-checked 16S rRNA gene database and

511 workbench compatible with ARB. Applied and Environmental Microbiology, 72(7),

512 5069-5072. doi:10.1128/AEM.03006-05

513 Dormann, C. F., Bobrowski, M., Dehling, D. M., Harris, D. J., Hartig, F., Lischke, H., ... 
514 Kraan, C. (2018). Biotic interactions in species distribution modelling: 10 questions to

515 guide interpretation and avoid false conclusions. Global Ecology and Biogeography.

516 doi:10.1111/geb.12759

517 Edgar, R. C. (2010). Search and clustering orders of magnitude faster than BLAST.

518 Bioinformatics, 26(19), 2460-2461. doi:10.1093/bioinformatics/btq461

519 Evans, S., Martiny, J. B. H., \& Allison, S. D. (2017). Effects of dispersal and selection on

520 stochastic assembly in microbial communities. The ISME Journal, 11(1), 176-185.

$521 \quad$ doi:10.1038/ismej.2016.96

522 Faust, K., Sathirapongsasuti, J. F., Izard, J., Segata, N., Gevers, D., Raes, J., \& Huttenhower,

523 C. (2012). Microbial co-occurrence relationships in the human microbiome. PLoS

524 Computational Biology, 8(7), e1002606. doi:10.1371/journal.pcbi.1002606

525 Ganz, H. H., Doroud, L., Firl, A. J., Hird, S. M., Eisen, J. A., \& Boyce, W. M. (2017).

526 Community-level differences in the microbiome of healthy wild mallards and those

527 infected by Influenza A riruses. MSystems, 2(1), e00188-16.

528 doi:10.1128/mSystems.00188-16

529 Gohl, D. M., Vangay, P., Garbe, J., MacLean, A., Hauge, A., Becker, A., ... Beckman, K. B.

530 (2016). Systematic improvement of amplicon marker gene methods for increased

531 accuracy in microbiome studies. Nature Biotechnology, 34(9), 942-949.

532 doi:10.1038/nbt.3601

533 Hanson, C. A., Fuhrman, J. A., Horner-Devine, M. C., \& Martiny, J. B. H. (2012). Beyond

534 biogeographic patterns: processes shaping the microbial landscape. Nature Reviews

$535 \quad$ Microbiology, 10(7), 497-506. doi:10.1038/nrmicro2795

536 Harris, D. J. (2016). Inferring species interactions from co-occurrence data with Markov

537 networks. Ecology, 97(12), 3308-3314. doi:10.1002/ecy.1605

538 Henderson, G., Cox, F., Ganesh, S., Jonker, A., Young, W., Global Rumen Census 
539 Collaborators, G. R. C., \& Janssen, P. H. (2015). Rumen microbial community

540 composition varies with diet and host, but a core microbiome is found across a wide

541 geographical range. Scientific Reports, 5, 14567. doi:10.1038/srep14567

542 Herren, C. M., \& McMahon, K. D. (2018). Keystone taxa predict compositional change in

543 microbial communities. Environmental Microbiology, 20(6), 2207-2217.

$544 \quad$ doi:10.1111/1462-2920.14257

545 Hofman-Kamińska, E., Bocherens, H., Borowik, T., Drucker, D. G., \& Kowalczyk, R.

546 (2018). Stable isotope signatures of large herbivore foraging habitats across Europe.

547 PLOS ONE, 13(1), e0190723. doi:10.1371/journal.pone.0190723

548 Hooper, L. V., Littman, D. R., \& Macpherson, A. J. (2012). Interactions between the

549 microbiota and the immune system. Science, 336(6086), 1268-1273.

550 doi: $10.1126 /$ science. 1223490

551 Hundertmark, K. J., Bowyer, R. T., Shields, G. F., \& Schwartz, C. C. (2003). Mitochondrial

552 phylogeography of moose (Alces alces) in North America. Journal of Mammalogy,

553 84(2), 718-728. doi:10.1644/1545-1542(2003)084<0718:MPOMAA>2.0.CO;2

554 Ishaq, S. L., Sundset, M. A., Crouse, J., \& Wright, A.-D. G. (2015). High-throughput DNA

555 sequencing of the moose rumen from different geographical locations reveals a core

556 ruminal methanogenic archaeal diversity and a differential ciliate protozoal diversity.

557 Microbial Genomics, 1(4), e000034. doi:10.1099/mgen.0.000034

558 Ishaq, S. L., \& Wright, A.-D. G. (2012). Insight into the bacterial gut microbiome of the

$559 \quad$ North American moose (Alces alces). BMC Microbiology, 12(1), 212.

560 doi:10.1186/1471-2180-12-212

561 Ishaq, S. L., \& Wright, A. D. (2014). High-throughput DNA sequencing of the ruminal

562 bacteria from moose (Alces alces) in Vermont, Alaska, and Norway. Microbial Ecology,

563 68(2), 185-195. doi:10.1007/s00248-014-0399-0 
564 Jani, A. J., \& Briggs, C. J. (2014). The pathogen Batrachochytrium dendrobatidis disturbs the

565 frog skin microbiome during a natural epidemic and experimental infection.

$566 \quad$ Proceedings of the National Academy of Sciences, 111(47), E5049-E5058.

567 doi:10.1073/pnas.1412752111

568 Jani, A. J., \& Briggs, C. J. (2018). Host and aquatic environment shape the amphibian skin 569 microbiome but effects on downstream resistance to the pathogen Batrachochytrium

$570 \quad$ dendrobatidis are variable. Frontiers in Microbiology, 9(MAR), 487.

$571 \quad$ doi:10.3389/fmicb.2018.00487

572 Kammann, E. E., \& Wand, M. P. (2003). Geoadditive models. Journal of the Royal

$573 \quad$ Statistical Society: Series C (Applied Statistics), 52(1), 1-18. doi:10.1111/1467-

$574 \quad 9876.00385$

575 Kanehisa, M., Sato, Y., Kawashima, M., Furumichi, M., \& Tanabe, M. (2016). KEGG as a

576 reference resource for gene and protein annotation. Nucleic Acids Research, 44(D1),

577 D457-D462. doi:10.1093/nar/gkv1070

578 Kohl, K. D., Varner, J., Wilkening, J. L., \& Dearing, M. D. (2018). Gut microbial

579 communities of American pikas ( Ochotona princeps ): Evidence for phylosymbiosis

580 and adaptations to novel diets. Journal of Animal Ecology, 87(2), 323-330.

$581 \quad$ doi:10.1111/1365-2656.12692

582 Langille, M. G. I., Zaneveld, J., Caporaso, J. G., McDonald, D., Knights, D., Reyes, J. A., ...

583 Huttenhower, C. (2013). Predictive functional profiling of microbial communities using

584 16S rRNA marker gene sequences. Nature Biotechnology, 31(9), 814-821.

585 doi:10.1038/nbt.2676

586 Le Borgne, H., Hébert, C., Dupuch, A., Bichet, O., Pinaud, D., \& Fortin, D. (2018). Temporal

587 dynamics in animal community assembly during post-logging succession in boreal

588 forest. PLOS ONE, 13(9), e0204445. doi:10.1371/journal.pone.0204445 
589 Lee, J. D., \& Hastie, T. J. (2015). Learning the structure of mixed graphical models. Journal 590 of Computational and Graphical Statistics, 24(1), 230-253.

591 doi:10.1080/10618600.2014.900500

592 Lenarz, M. S. (2009). 2009 Aerial Moose Survey. St Paul, USA.

593 Ley, R. E., Hamady, M., Lozupone, C., Turnbaugh, P. J., Ramey, R. R., Bircher, J. S., ...

594 Gordon, J. I. (2008). Evolution of mammals and their gut microbes. Science, 320(5883),

595 1647-1651. doi:10.1126/science.1155725

596 Masella, A. P., Bartram, A. K., Truszkowski, J. M., Brown, D. G., \& Neufeld, J. D. (2012).

597 PANDAseq: paired-end assembler for illumina sequences. BMC Bioinformatics, 13(1),

598 31. doi:10.1186/1471-2105-13-31

599 McKenney, E. A., Williamson, L., Yoder, A. D., Rawls, J. F., Bilbo, S. D., \& Parker, W.

600 (2015). Alteration of the rat cecal microbiome during colonization with the helminth

601 Hymenolepis diminuta. Gut Microbes, 6(3), 182-193.

602 doi:10.1080/19490976.2015.1047128

603 Methé, B. A., Nelson, K. E., Pop, M., Creasy, H. H., Giglio, M. G., Huttenhower, C., ...

604 White, O. (2012). A framework for human microbiome research. Nature, 486(7402),

605 215-221. doi:10.1038/nature11209

606 Moeller, A. H., Peeters, M., Ayouba, A., Ngole, E. M., Esteban, A., Hahn, B. H., \& Ochman,

607 H. (2015). Stability of the gorilla microbiome despite simian immunodeficiency virus

608 infection. Molecular Ecology, 24(3), 690-697. doi:10.1111/mec.13057

609 Moeller, A. H., Suzuki, T. A., Lin, D., Lacey, E. A., Wasser, S. K., \& Nachman, M. W.

610 (2017). Dispersal limitation promotes the diversification of the mammalian gut

611 microbiota. Proceedings of the National Academy of Sciences of the United States of

$612 \quad$ America, 114(52), 13768-13773. doi:10.1073/pnas.1700122114

613 Mshelia, E. S., Adamu, L., Wakil, Y., Turaki, U. A., Gulani, I. A., \& Musa, J. (2018). The 
association between gut microbiome, sex, age and body condition scores of horses in

615 Maiduguri and its environs. Microbial Pathogenesis, 118, 81-86.

616 doi:10.1016/j.micpath.2018.03.018

617 Muegge, B. D., Kuczynski, J., Knights, D., Clemente, J. C., González, A., Fontana, L., ...

618 Gordon, J. I. (2011). Diet drives convergence in gut microbiome functions across

619 mammalian phylogeny and within humans. Science, 332(6032), 970-4.

620 doi:10.1126/science.1198719

621 Murray, D. L., Cox, E. W., Ballard, W. B., Whitlaw, H. A., Lenarz, M. S., Custer, T. W., ...

622 Fuller, T. K. (2006). Pathogens, nutritional deficiency, and climate influences on a

623 declining moose population. Wildlife Monographs, 166(1), 1-30. doi:10.2193/0084-

$624 \quad$ 0173(2006)166[1:PNDACI $] 2.0 . \mathrm{CO} ; 2$

625 Näpflin, K., \& Schmid-Hempel, P. (2018). Host effects on microbiota community assembly.

626 Journal of Animal Ecology, 87(2), 331-340. doi:10.1111/1365-2656.12768

627 Nemergut, D. R., Schmidt, S. K., Fukami, T., O’Neill, S. P., Bilinski, T. M., Stanish, L. F.,

628 ... Ferrenberg, S. (2013). Patterns and processes of microbial community assembly.

629 Microbiology and Molecular Biology Reviews $\square: M M B R, 77(3)$, 342-56.

630 doi:10.1128/MMBR.00051-12

631 Oksanen, J., Blanchet, F. G., Kindt, R., Legendre, P., Minchin, P. R., O’Hara, R. B., ...

632 Wagner, H. (2013). Package 'vegan.’ R Package Ver. 2.0-8, 254.

633 Ovaskainen, O., Abrego, N., Halme, P., \& Dunson, D. (2016). Using latent variable models

634 to identify large networks of species-to-species associations at different spatial scales.

635 Methods in Ecology and Evolution, 7(5), 549-555. doi:10.1111/2041-210X.12501

636 Ovaskainen, O., Tikhonov, G., Norberg, A., Guillaume Blanchet, F., Duan, L., Dunson, D.,

637 ... Abrego, N. (2017). How to make more out of community data? A conceptual

638 framework and its implementation as models and software. Ecology Letters, 20(5), 561- 
576. doi:10.1111/ele.12757

640 Severud, W. (2017). Assessing calf survival and the quantitative impact of reproductive

641 success on the declining moose (Alces alces) population in northeastern Minnesota.

$642 \quad$ University of Minnesota. Retrieved from

643 https://conservancy.umn.edu/handle/11299/191446

644 Svartström, O., Alneberg, J., Terrapon, N., Lombard, V., De Bruijn, I., Malmsten, J., ...

645 Andersson, A. F. (2017). Ninety-nine de novo assembled genomes from the moose

646 (Alces alces) rumen microbiome provide new insights into microbial plant biomass

647 degradation. ISME Journal, 11(11), 2538-2551. doi:10.1038/ismej.2017.108

648 Vellend, M., Srivastava, D. S., Anderson, K. M., Brown, C. D., Jankowski, J. E., Kleynhans,

649 E. J., ... Xue, X. (2014). Assessing the relative importance of neutral stochasticity in

650 ecological communities. Oikos, 123(12), 1420-1430. doi:10.1111/oik.01493

651 Wood, S. N. (2003). Thin plate regression splines. Journal of the Royal Statistical Society:

652 Series B (Statistical Methodology), 65(1), 95-114. doi:10.1111/1467-9868.00374

653 Wünschmann, A., Armien, A. G., Butler, E., Schrage, M., Stromberg, B., Bender, J. B., ...

654 Carstensen, M. (2015). Necropsy findings in 62 opportunistically collected free-ranging

655 moose (Alces alces) from Minnesota, USA (2003-13). Journal of Wildlife Diseases,

$656 \quad 51(1), 157-165$. doi:10.7589/2014-02-037

657 Zelezniak, A., Andrejev, S., Ponomarova, O., Mende, D. R., Bork, P., \& Patil, K. R. (2015).

658 Metabolic dependencies drive species co-occurrence in diverse microbial communities.

659 Proceedings of the National Academy of Sciences of the United States of America,

660 112(20), 6449-54. doi:10.1073/pnas.1421834112

661 Zhou, J., \& Ning, D. (2017). Stochastic community assembly: Does it matter in Microbial

662 Ecology? Microbiology and Molecular Biology Reviews $\square:$ MMBR, 81(4), e00002-17.

663 doi:10.1128/MMBR.00002-17 
bioRxiv preprint doi: https://doi.org/10.1101/514604; this version posted January 9,2019 . The copyright holder for this preprint (which was not certified by peer review) is the author/funder, who has granted bioRxiv a license to display the preprint in perpetuity. It is made available under aCC-BY-NC-ND 4.0 International license.

\section{4}

